\title{
Translation and validation of the Indian Takayasu clinical activity score (ITAS2010) for the Brazilian Portuguese language
}

\author{
Scheila Fritsch'1 ${ }^{1}$ Rafaela Martinez Copes ${ }^{1}$, Bruna Savioli ${ }^{2}$, Mariana Freitas de Aguiar ${ }^{2}$, Rozana Mesquita Ciconelli ${ }^{2}$,
} Valderílio Feijó Azevedo ${ }^{1}$ and Alexandre W. S. de Souza ${ }^{2^{*}}$ (D)

\begin{abstract}
Background: The Indian Takayasu Clinical Activity Score (ITAS2010) was developed in 2010 as an assessment tool for disease activity in patients with Takayasu arteritis (TA). It has since been widely used in different studies and in clinical practice for the management of patients with TA. The present study aims to translate the ITAS2010 into Brazilian Portuguese language and to validate it for use in clinical practice in Brazil.

Methods: For this cross-sectional study, the ITAS2010 was translated in accordance with the guidelines described by Beaton et al. and then applied with 27 patients with TA on three assessments by two rheumatologists working independently. To measure interrater agreement, the assessments were performed on the same day within approximately 1 hour. One of the rheumatologists performed a second evaluation of patients with TA within 7 to 14 days to measure intrarater agreement.

Results: The correlation coefficient for the ITAS2010 score between the two raters was high $(r=0.916 ; p<0.0001)$, as well as the intraclass correlation coefficient (ICC) [0.918 with a 95\% confidence interval (95CI): 0.828-0.962]. The correlation coefficient and the ICC for intrarater agreement were moderate for ITAS2010 $(r=0.633 ; p<0.0001$ and ICC = 0.594; 95Cl: 0.292-0.790). The ITAS2010 at baseline was compared with the physician's global assessment (PGA) and with Kerr's criteria for detecting disease activity in TA. Higher ITAS2010 scores were observed in patients with active and grumbling/persistent disease than in those presenting inactive disease according to the PGA [1.5 $(0.0-3.0)$ vs. $0.0(0.0-0.0) ; p=0.0025]$. Patients with active disease according to the Kerr's criteria had also higher ITAS2010 scores than those considered in remission [3.0 (3.0-7.0) vs. $0.0(0.0-0.0) ; p=0.0068]$.
\end{abstract}

Conclusions: The Brazilian Portuguese version of the ITAS2010 is a valid and reproducible tool for the assessment of disease activity in TA and it is an additional tool for the routine evaluation of Brazilian patients with TA.

Keywords: Large-vessel vasculitis, Takayasu arteritis, Disease activity, Outcome measures

\section{Background}

Takayasu arteritis (TA) is a large-vessel vasculitis that affects the aorta, its major branches, and pulmonary arteries. The inflammatory process in TA affects all layers of the arterial wall and leads to concentric thickening, stenosis, occlusion, dilation and/or aneurysm formation [1]. TA usually follows a relapse-remitting course, but the assessment of disease activity in TA is often difficult, because active

\footnotetext{
* Correspondence: alexandre_wagner@uol.com.br

${ }^{2}$ Rheumatology Division, Universidade Federal de São Paulo, Escola Paulista de Medicina (Unifesp-EPM), rua Botucatu, 740, São Paulo, SP 04023-900, Brazil Full list of author information is available at the end of the article
}

vasculitic process may lead to vascular lesions without overt signs and symptoms; indeed, new angiographic lesions have been reported to develop in previously unaffected vascular territories even in asymptomatic patients [2]. Disease activity in TA has been assessed in clinical practice by the presence of constitutional symptoms, new vascular complaints (e.g., claudication and carotidynia), and new findings on vascular examination (e.g., bruits and pulse loss), alterations in acute phase reactants, and on vascular imaging studies [1]. However, it is difficult to differentiate between active disease and permanent vascular damage in TA. Valid tools to assess disease activity in TA are needed not only in

(C) The Author(s). 2019 Open Access This article is distributed under the terms of the Creative Commons Attribution 4.0 International License (http://creativecommons.org/licenses/by/4.0/), which permits unrestricted use, distribution, and 
clinical practice but also in clinical trials that evaluate therapeutic agents for TA $[3,4]$.

In a prospective study published in 1994, 60 patients with TA were followed up for 5.3 years at the National Institutes of Health (NIH). Kerr's criteria or the NIH criteria were then proposed to assess disease activity in TA based on the onset of new disease manifestations, altered erythrocyte sedimentation rate (ESR) and new angiographic lesions [5]. These criteria are only qualitative and they have been widely used in clinical practice and in studies evaluating patients with TA [6]. Kerr's criteria, however, have proven insensitive to disease activity in TA, since $61 \%$ of asymptomatic patients at follow-up had developed angiographic abnormalities in previously unaffected vascular territories, and active arterial inflammation was observed in $44 \%$ of patients with TA who were thought to be in remission and underwent arterial bypass surgery [5].

The Birmingham Vasculitis Activity Score (BVAS) is a tool developed to assess disease activity in systemic vasculitides. Three versions of the BVAS score and the BVAS-WG (BVAS for Wegener's granulomatosis) are now available for use in clinical practice. The third version of the BVAS score comprises 56 items for vasculitic manifestations in 9 organs and systems $[7,8]$. BVAS items, however, are not as suitable to assess disease activity in large-vessel vasculitides as in small- and medium-vessel vasculitides [6]. The Disease Extent Index for Takayasu's Arteritis (DEI.Tak) was derived from the BVAS to assess disease activity in TA, it has higher weights for scoring vascular items and it was designed to evaluate the disease extent in TA, including both disease activity and damage [9]. Regarding disease activity in TA, DEI.TAk was shown to differentiate poorly between disease activity and remission [10].

The Indian Takayasu Activity Score (ITAS2010) is the first valid assessment tool to quantify disease activity in TA. The ITAS2010 contains 44 features, 33 of which pertain to the cardiovascular system. Seven key items are weighted to score 2 . Only new manifestations occurring in the previous 3 months are to be recorded on the ITAS2010 form. The composite ITAS-A, combining the ITAS2010 score with acute phase reactants such as ESR and C-reactive protein (CRP), may also be used to assess patients with TA [9]. ITAS2010 is sensitive to change and it has already been used in different studies to assess disease activity in TA, including an open-label study of mycophenolate mofetil in TA and in a large cohort study performed in India that assessed longterm outcomes in TA [11-17]. The present study aims to translate the ITAS2010 into Brazilian Portuguese and to validate it for use with Portuguese-speaking patients with TA.

\section{Methods}

\section{Patients with Takayasu arteritis}

Authorization for this prospective cross-sectional study was obtained from one of the investigators involved in the development of the ITAS2010, Dr. P. A. Bacon. It had a cross-sectional design. The research protocol was approved by the Institutional Review Boards and complied with the Declaration of Helsinki. All participants of the study provided a written informed consent. Consecutive TA patients under regular follow-up were included in the study if they were above 18 years of age and if they fulfilled the 1990 American College of Rheumatology criteria for TA or Ishikawa's diagnostic criteria for TA modified by Sharma et al. [18, 19].

\section{Translation of the ITAS2010}

The translation and cross-cultural adaptation of the ITAS2010 followed the guidelines described by Beaton et al [20]. Initially, the original version of the ITAS2010 and its glossary were translated to the Portuguese language by two independent Brazilian certified translators to create the versions $\mathrm{T} 1$ and $\mathrm{T} 2$. These versions were reviewed and compared by the investigators and translators to produce version T12 of the instrument (Fig. 1) and its glossary (Additional file 1). Two other translators, native speakers of English who were fluent in Portuguese and blind to the original documents, translated the version T12 from Portuguese into English. This back-translation process resulted in two versions (BT1 and BT2) which were compared with the original version of the ITAS2010.

\section{Validation of the ITAS2010}

Two rheumatologists (SF and RMC), experienced with the management of patients with TA tested the reliability and validity of the T12 Brazilian Portuguese version of the ITAS2010. The clinical assessment of TA patients was standardized in accordance with the ITAS2010 form, and included history taking, physical examination, and a review of the patient's medical records. For interrater variability to be tested, the ITAS2010 was applied at baseline by SF once the patient had provided the written informed consent and, approximately 1 hour later, by the other investigator (RMC). For intrarater variability to be tested, the ITAS2010 was applied at a second visit within 7 to 14 days after baseline. The rheumatologists were blinded to ITAS2010 scores obtained by each other. During history taking, symptoms were recorded only if their onset had occurred earlier than 3 months.

The physical examination of TA patients was standardized and included auscultation of heart and vascular murmurs (carotid, aortic, subclavian and renal arteries murmurs) and the palpation of peripheral pulses bilaterally on carotid, subclavian, brachial, radial, femoral, popliteal, dorsal pedialis and posterior tibial arteries. Blood pressure (BP) was measured in the four limbs. Systolic blood pressure differences between limbs were considered significant when greater than 10 mmHg. Physical examination changes were recorded in the ITAS2010 only if they had been absent ( $>3$ months ago). 


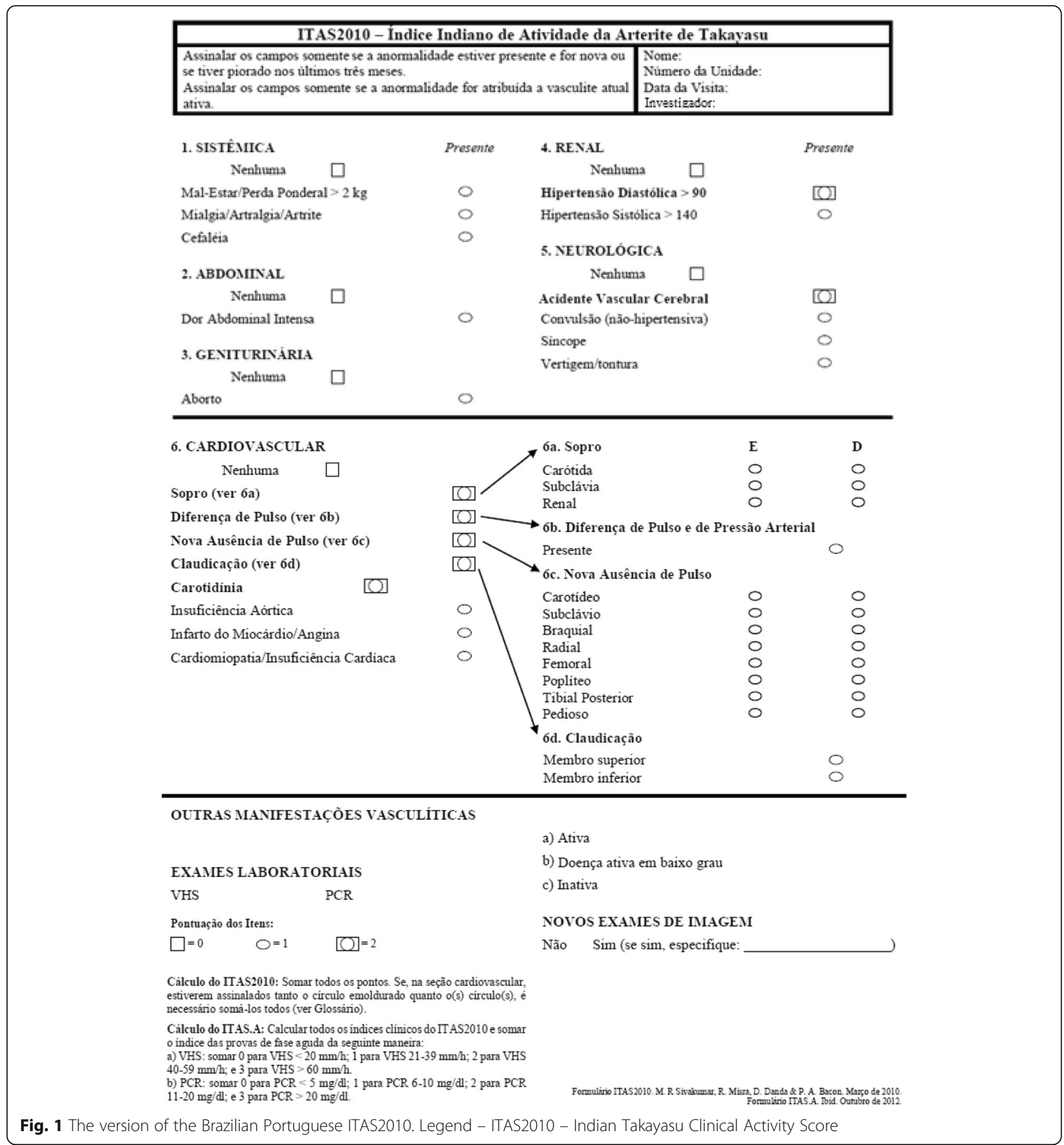

ESR and CRP results were analyzed for the ITAS. A if the tests had been performed within the last 30 days before patient's initial assessment. The physician's global assessment (PGA) was used as a gold standard to ascertain disease activity in TA as it included clinical, laboratory and imaging data as well as recent change in therapy (checked in medical records and/or reported by TA patients). The PGA differentiated three categories: active disease, grumbling/persistent disease, and inactive disease. Only after the second assessment by SF, data from TA patients were computed. The convergent validity of the Brazilian Portuguese version of the ITAS2010 was analyzed by comparing ITAS2010 results with the disease activity ascertained by the PGA and Kerr's criteria [5, 9].

\section{Statistical analysis}

Statistical analysis was performed using the IBM SPSS Statistics for Windows, version 20.0 (Armonk, USA) and 
graphs were built using the GraphPad Prism version 5.0. Categorical data were presented as absolute number and percentage. The Shapiro Wilk test was used as a normality test and continuous variables were presented as mean and standard deviation or as median and interquartile range. To evaluate the reproducibility of the Brazilian Portuguese version of the ITAS2010, the assessments at baseline by both rheumatologists (SF and RMC) and the first and second assessments by SF were analyzed. The Pearson's correlation coefficient and the intraclass correlation coefficient (ICC) were used to evaluate the reproducibility of the ITAS2010. Convergent validity was analyzed by comparing ITAS2010 and ITAS. A results with PGA using Mann Whitney's test. The significance level accepted was $5 \%(p<0.05)$.

\section{Results}

\section{Demographic features of TA patients}

A total of 42 TA patients were initially invited to participate in the study, 15 patients had to be excluded. Twenty-seven TA patients who complied with research protocol were included in the final analysis. Figure 2 shows the flow of participants through the study. Females comprised $92.6 \%$ of patients and the mean age at study was $43.8 \pm 12.9$ years.

\section{Reliability of the ITAS2010}

The analysis of the intra-rater reproducibility of the ITAS2010 showed a significant correlation between SF assessments at baseline and SF 2 weeks later $(r=0.633$; $p<0.0001)$ and a moderate reproducibility for SF at T0 and SF at T1 [ICC $=0.594$ (95CI: $0.292-0.790$ ); $p<$ $0.0001]$. Nonetheless, the interrater reproducibility of the

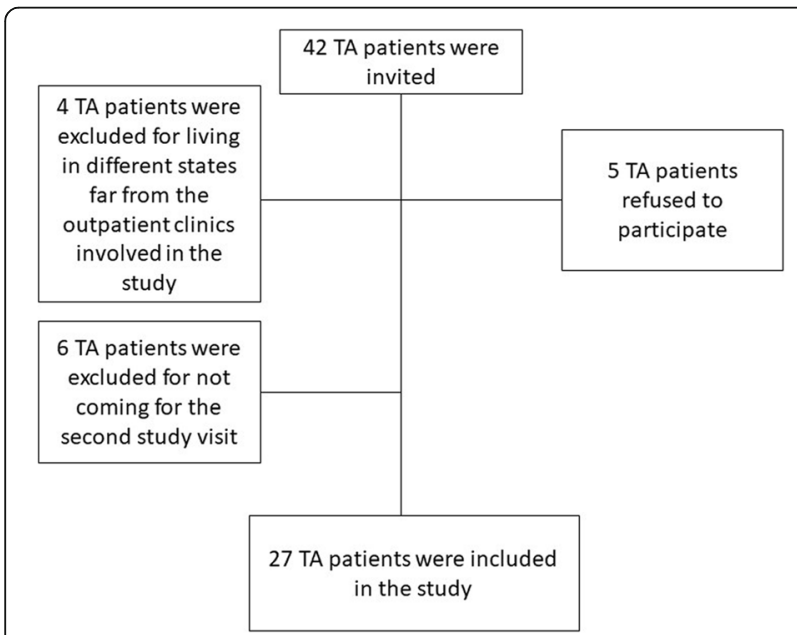

Fig. 2 Flowchart showing the recruitment and exclusion of Takayasu arteritis patients in the study. Legend - Fifteen TA patients were excluded from the study, six of them did not comply with study's protocol. TA - Takayasu arteritis
ITAS2010 applied at baseline was very strong for SF and RMC assessments with a high correlation $r=0.916$; $(p<$ 0.0001 ) and ICC $=0.918$ (95CI: $0.828-0.962 ; p<0.0001$ ).

\section{Convergent validity of the ITAS2010}

In the analysis of the convergent validity, ITAS2010 scores were compared with two other tools to assess disease activity in TA: the PGA and Kerr's criteria. The PGA was split in two categories: the first included active disease and grumbling/persistent disease $(N=8)$ and the other category included patients with inactive disease $(N=16)$. Only three TA patients presented active disease and 21 were in remission according to the Kerr's criteria. The median ITAS2010 score was significantly higher in TA patients with active disease and grumbling/persistent disease than in those with inactive disease according to the PGA [1.5 (0.0-3.0) vs. $0.0(0.0-0.0) ; p=0.0025]$ and also higher in patients with active disease by Kerr's criteria than in those considered to be in remission [3.0 $(3.0-7.0)$ vs. $0.0(0.0-0.0) ; p=0.0068$ ] (Fig. 3). When acute phase reactants were added, patients with active disease and grumbling/persistent disease had higher ESR ITAS. A scores than those with inactive disease [3.0 $(2.0-6.0)$ vs. $1.0(0.0-2.0) ; p=0.0123]$, whereas no significant differences were found between CRP ITAS.A scores active disease and grumbling/persistent disease and CRP ITAS. A scores for inactive disease [1.5 (0.0$4.0)$ vs. $0.0(0.0-1.0) ; p=0.0950]$. Moreover, the median ESR ITAS.A was significantly higher in TA patients presenting active disease according to Kerr's criteria than in those in remission [6.0 (3.0-9.0) vs. $1.0(0.0-2.0) ; p=$ 0.016]. Statistical analysis could not be performed for CRP ITAS. A because of the small number of patients with active TA and CRP results at study.

\section{Discussion}

In this study, the ITAS2010 was translated into Brazilian Portuguese and validated as an assessment tool for disease activity in Brazilian patients with TA, though we believe that it may be easily and correctly applied in different Portuguese-speaking countries. The ITAS2010 presented a very high interobserver reproducibility with a moderate intraobserver reliability. Despite the relatively low number of patients with TA presenting active disease, the Brazilian version of the ITAS2010 was able to discriminate disease activity from the remission state according to the PGA and Kerr's criteria. The Brazilian Portuguese version of the ITAS2010 is therefore suitable for use in clinical practice.

The Brazilian Portuguese version of the ITAS2010 is a reliable tool to evaluate disease activity in TA. Its interrater reproducibility was extremely high when the assessment was performed on the same day by two different physicians as demonstrated by the Pearson's correlation coefficient $(r=0.916)$ and the ICC $(0.918)$. The latter had 


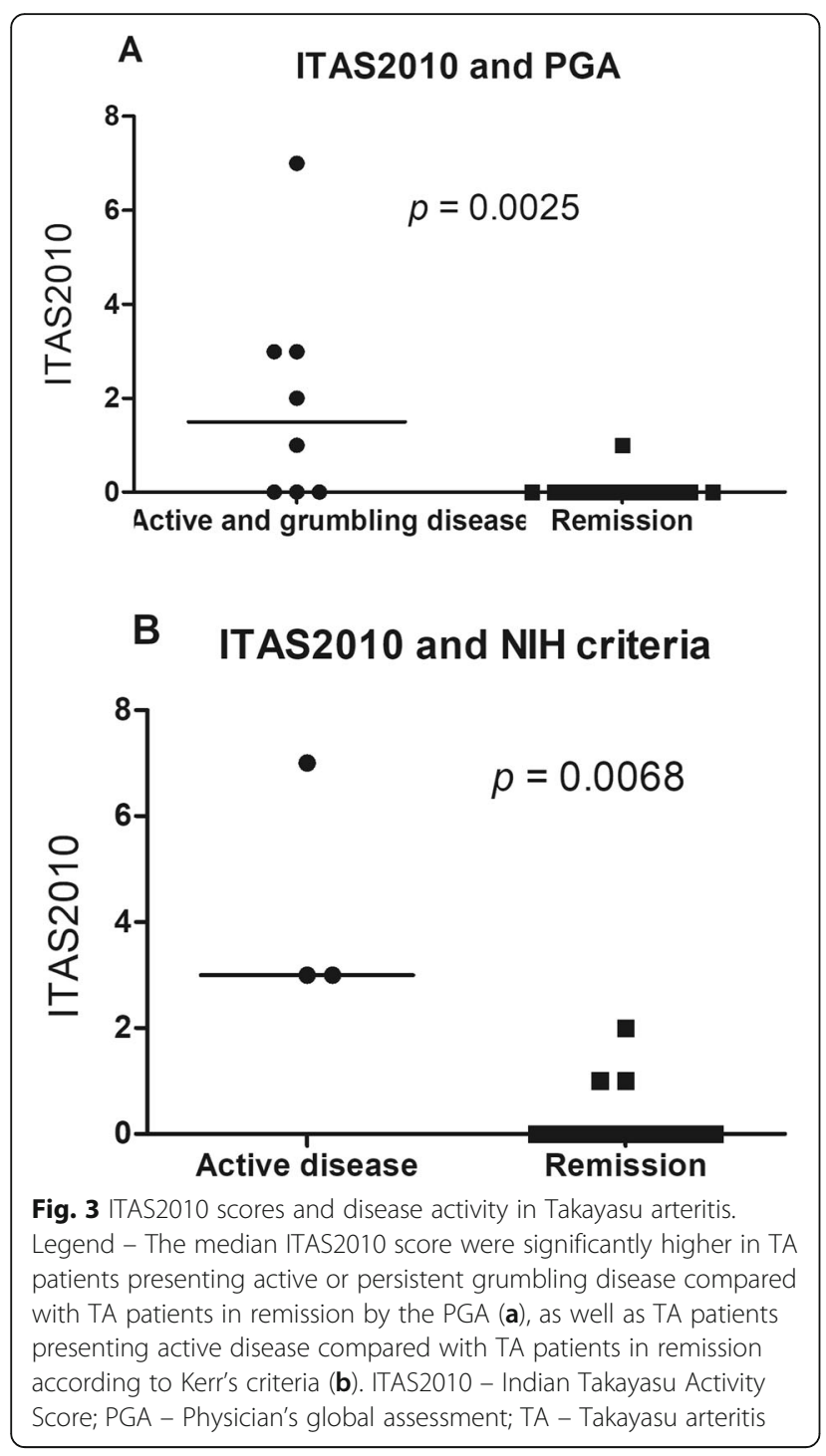

also a narrow confidence interval (0.828-0.962). These data are quite robust, indicating that when the Brazilian Portuguese version of the ITAS2010 was applied by two different observers within approximately 1 hour, both measurements presented similar results.

Unfortunately, the intrarater reliability of the Brazilian Portuguese version of the ITAS2010 was only moderate for both Pearson's coefficient $(r=0.633)$ and the ICC (0.594) with a wide CI (0.292-0.790). However, we do not believe that this finding is due to shortcomings of the Brazilian Portuguese version of the ITAS2010 itself. The time lapse between the baseline and follow-up evaluations by SF (i.e. 7 to 14 days) may have accounted for changes in presenting complaints and in vascular examination, since all patients with TA presenting active disease at baseline received high-dose glucocorticoids and changed their immunosuppressive or biologic therapy at the attending physician's discretion. Indeed, it has been shown that the ITAS2010 score is sensitive to detect changes in disease manifestations as a consequence of therapy changes in TA [9].

Regarding the convergent validity, the analysis between ITAS2010 and the NIH criteria was hampered by the fact that there were only three patients with active TA according to Kerr's criteria. Convergent validity was better established for the ITAS2010/ITAS.A and the PGA. In agreement with results described by Misra et al. [9], the median ITAS2010 score was significantly higher in patients with active disease than in patients in remission, according to the both instruments, the PGA and the NIH criteria. Only CRP ITAS.A was not different between patients with active/grumbling TA and those in remission. These findings suggest that the Brazilian Portuguese version of the ITAS2010 is a valid tool to differentiate between activity and remission, when compared with other outcome measures such as the PGA and Kerr's criteria in TA.

This study has some limitations. First, the relatively small sample of patients with TA included in the study, especially the number of patients with TA presenting active disease, hampered the assessment of the convergent validity. In the study that had validated the ITAS2010, the vast majority of patients with TA presented active disease when they were evaluated (i.e., 132 patients with clinically active disease as opposed to 33 patients in remission and 11 with grumbling disease) [9]. The small number of participants in our study could have interfered with the validation of ITAS2010, but reliability and convergent validity were analyzed and, with statisticaly significant results, corroborated the validation. Two other important sources of bias in this study are worth mentioning: the fact that patients had to remember if the onset of their symptoms had occurred in less than 3 months, and the fact that the measurement of ESR and CRP had to be collected from the patient's medical records when they could not be performed at baseline.

\section{Conclusion}

The Brazilian Portuguese version of the ITAS2010 is reproducible and sensitive enough to detect disease activity in TA consistent with the PGA and Kerr's criteria. The Brazilian Portuguese version of the ITAS2010 and the ESR/CRP ITAS. A could therefore facilitate disease assessment and therapeutic decisions in the routine evaluation of patients with TA in Brazil and allow the standardization of evaluation of TA in future studies.

\section{Supplementary information}

Supplementary information accompanies this paper at https://doi.org/10. 1186/s42358-019-0087-3.

Additional file 1. Supplementary glossary for the ITAS2010 and ITAS.A 


\section{Abbreviations}

BT: Back translation; BVAS: Birmingham Vasculitis Activity Score;

Cl: Confidence interval; CRP: C-reactive protein; DEl.Tak: Disease extent index for Takayasu's arteritis; ESR: Erythrocyte sedimentation rate; ICC: Intraclass correlation coefficient; ITAS.A: Indian Takayasu Clinical Activity Score - Acute phase reactants; ITAS2010: Indian Takayasu Clinical Activity Score; NIH: National Institutes of Health; PGA: Physician's global assessment; TA: Takayasu arteritis

\section{Acknowledgements}

The authors thank the following contributors for this project: Nicholas Anthony Williams (certified English-Portuguese translator), responsible for the Portuguese version - T1; Bruno lochio Mori, certified English-Portuguese translator, responsible for the translated Portuguese version - T2; Neil Dallas, certified translator, native of English speaking country, fluent in Portuguese, responsible for the translated version in English - BT1; Todd Price, certified translator, native of English speaking country, fluent in Portuguese, responsible for the translated version in English - BT2.

\section{Authors' contributions}

SF contributed to the study design, evaluated the study participants, collected data from medical records and drafted the manuscript. RMC1 evaluated the study participants, collected data from medical records, helped with the interpretation of data and revised the manuscript. MFA and BS helped the assessment of study participants, collected data from medical records, helped with the interpretation of data and revised the manuscript. RMC2 contributed to the study design, helped with the interpretation of data and revised the manuscript. VFA contributed to the study design, helped with the interpretation of data and revised the manuscript. AWSS conceived the study, contributed to the study design, helped with the interpretation of results and revised draft version of the manuscript. All authors read and approved the manuscript.

\section{Funding}

This study has not received any kind of financial support or grants.

\section{Availability of data and materials}

The database used for this study are available from the corresponding author upon reasonable request.

\section{Ethics approval and consent to participate}

The research protocol was approved by the Institutional Review Boards of both institutions involved in the study: Comitê de Ética em Pesquisa da UNIFESP-EPM and by the Comitê de Ética em Pesquisa from HC-UFPR. This study complied with the Declaration of Helsinki and all participants gave written informed consent.

\section{Consent for publication}

All authors are aware of the content of this article and provided consent for publication in Advances in Rheumatology.

\section{Competing interests}

The authors declare that they have no competing interests.

\section{Author details}

'Rheumatology Division, Universidade Federal do Paraná (UFPR), rua Padre Camargo, 280. Alto da Glória, Curitiba, PR 80060-240, Brazil. ${ }^{2}$ Rheumatology Division, Universidade Federal de São Paulo, Escola Paulista de Medicina (Unifesp-EPM), rua Botucatu, 740, São Paulo, SP 04023-900, Brazil.

\section{Received: 6 February 2019 Accepted: 26 September 2019}

\section{Published online: 16 October 2019}

\section{References}

1. Mason JC. Takayasu arteritisadvances in diagnosis and management. Nat Rev Rheumatol. 2010;6(7):406-15. https://doi.org/10.1038/nrrheum.2010.82.

2. Maksimowicz-McKinnon K, Hoffman GS. Takayasu arteritis: what is the longterm prognosis? Rheum Dis Clin N Am. 2007;33(4):777-86.

3. Direskeneli H. Clinical assessment in Takayasu's arteritis: major challenges and controversies. Clin Exp Rheumatol. 2017;35(Suppl 1(1)):189-93.

4. Seyahi E. Takayasu arteritis: an update. Curr Opin Rheumatol. 2017;29(1):51-6.
5. Kerr GS, Hallahan CW, Giordano J, Leavitt RY, Fauci AS, Rottem M, et al. Takayasu arteritis. Ann Intern Med. 1994;120(11):919-29.

6. Direskeneli H, Aydin SZ, Merkel PA. Assessment of disease activity and progression in Takayasu's arteritis. Clin Exp Rheumatol. 2011;29(1 Suppl 64):S86-91.

7. Mukhtyar C, Lee R, Brown D, Carruthers D, Dasgupta B, Dubey S, et al. Modification and validation of the Birmingham Vasculitis activity score (version 3). Ann Rheum Dis. 2009;68(12):1827-32.

8. Stone JH, Hoffman GS, Merkel PA, Min YI, Uhlfelder ML, Hellmann DBSU, Allen NB, Davis JC, Spiera RF, Calabrese LH, Wigley FM, Maiden NVR, Niles $J$, Fye KH, McCune JW, St Clair EW. LRIN for, (INSSYS). The S of the SV. A disease-specific activity index for Wegener's granulomatosis: modification of the Birmingham Vasculitis activity score. International network for the study of the systemic Vasculitides (INSSYS). Arthritis Rheum. 2001;44(4):912-20.

9. Misra R, Danda D, Rajappa SM, Ghosh A, Gupta R, Mahendranath KM, et al. Development and initial validation of the Indian Takayasu clinical activity score (ITAS2010). Rheumatology (Oxford). 2013;52(10):1795-801.

10. Aydin SZ, Yilmaz N, Akar S, Aksu K, Kamali S, Yucel E, et al. Assessment of disease activity and progression in Takayasu's arteritis with disease extent index-Takayasu. Rheumatology (Oxford). 2010;49(10):1889-93.

11. Alibaz-Oner F, Yentur SP, Saruhan-Direskeneli G, Direskeneli H. Serum cytokine profiles in Takayasu's arteritis: search for biomarkers. Clin Exp Rheumatol. 2015;33(2 Suppl 89):S-32-5.

12. Misra DP, Chaurasia S, Misra R. Increased circulating Th17 cells, serum IL17A, and IL-23 in Takayasu arteritis. Autoimmune Dis. 2016;2016:7841718.

13. Goel R, Kabeerdoss J, Ram B, Prakash JAJ, Babji S, Nair A, et al. Serum cytokine profile in Asian Indian patients with Takayasu arteritis and its association with disease activity. Open Rheumatol J. 2017;11:23-9.

14. de Souza AWS, van der Geest KSM, Brouwer E, Pinheiro FAG, Oliveira ACD, Sato El, et al. High mobility group box 1 levels in large vessel vasculitis are not associated with disease activity but are influenced by age and statins. Arthritis Res Ther. 2015;17(1):158

15. Goel R, Danda D, Mathew J, Edwin N. Mycophenolate mofetil in Takayasu's arteritis. Clin Rheumatol. 2010;29(3):329-32.

16. Pulsatelli L, Boiardi L, Assirelli E, Pazzola G, Muratore F, Addimanda O, et al. Interleukin-6 and soluble interleukin-6 receptor are elevated in large-vessel vasculitis: a cross-sectional and longitudinal study. Clin Exp Rheumatol. 2017;35(Suppl 1(1)):102-10

17. Goel R, Danda D, Joseph G, Ravindran R, Kumar S, Jayaseelan VIL. P. B. long-term outcome of 251 patients with Takayasu arteritis on combination immunosuppressant therapy: single Centre experience from a large tertiary care teaching hospital in southern India. Semin Arthritis Rheum. 2018;47(5):718-26.

18. Arend WP, Michel BA, Bloch DA, Hunder GG, Calabrese LH, Edworthy SM, et al. The American College of Rheumatology 1990 criteria for the classification of Takayasu arteritis. Arthritis Rheum. 1990;33(8):1129-34.

19. Sharma BK, Jain S, Suri SNF. Diagnostic criteria for Takayasu arteritis. Int J Cardiol. 1996;54(Suppl):S141-7.

20. Beaton DE, Bombardier C, Guillemin F, Ferraz MB. Guidelines for the process of cross-cultural adaptation of self-report measures. Spine (Phila Pa 1976). 2000;25(24):3186-91.

\section{Publisher's Note}

Springer Nature remains neutral with regard to jurisdictional claims in published maps and institutional affiliations.
Ready to submit your research? Choose BMC and benefit from:
- fast, convenient online submission
- thorough peer review by experienced researchers in your field
- rapid publication on acceptance
- support for research data, including large and complex data types
- gold Open Access which fosters wider collaboration and increased citations
- maximum visibility for your research: over $100 \mathrm{M}$ website views per year
At BMC, research is always in progress.
Learn more biomedcentral.com/submissions 